\title{
Topotaxy relationships in the transformation phosgenite-cerussite
}

\author{
C.M. Pina ${ }^{\mathrm{a}, *}$, L. Fernández-Díaz ${ }^{\mathrm{a}}$, M. Prieto ${ }^{\mathrm{b}}$ \\ a Departamento de Cristalografia y Mineralogia, Universidad Complutense de Madrid, E-28040 Madrid, Spain \\ ${ }^{\mathrm{b}}$ Departamento de Geologia, Universidad de Oviedo (IUQOM), E-33005 Oviedo, Spain
}

\begin{abstract}
The crystallization of phosgenite $\left(\mathrm{Pb}_{2} \mathrm{Cl}_{2} \mathrm{CO}_{3}\right)$ and cerussite $\left(\mathrm{PbCO}_{3}\right)$ has been carried out by counter diffusion in a silica hydrogel at $25^{\circ} \mathrm{C}$. The crystallization of both phases is strongly controlled by the $\mathrm{pH}$ of the medium. Under certain conditions the physicochemical evolution of the system determines that phosgenite crystals become unstable and transform into cerussite. The transformation is structurally controlled and provides an interesting example of topotaxy. The orientational relationships are $\langle 110\rangle_{\mathrm{ph}}\left\|[001]_{\mathrm{cer}},\langle 110\rangle_{\mathrm{ph}}\right\|[100]_{\mathrm{cer}}$, and $(001)_{\mathrm{ph}} \|(010)_{\mathrm{cer}}$. In this paper, a study of the topotactic transformation and the crystal morphology is worked out. The topotactic relationships are interpreted on the ground of geometrical and structural considerations.
\end{abstract}

\section{Introduction}

Phosgenite $\left(\mathrm{Pb}_{2} \mathrm{Cl}_{2} \mathrm{CO}_{3}\right)$ and cerussite $\left(\mathrm{PbCO}_{3}\right)$ are two secondary lead minerals that form in supergenic deposits as an alteration product of galene and anglesite. The crystallization of phosgenite and cerussite is strongly controlled by the $\mathrm{pH}$ of the medium. Phosgenite crystallizes at $\mathrm{pH}$ values around 5 , while cerussite nucleation requires a basic $\mathrm{pH}[1]$. Transformation of phosgenite into cerussite has been frequently observed in nature [2] as a result of changes in $\mathrm{pH}$ and physicochemical conditions. This transformation seems to be controlled by a solventmediated dissolution-recrystallization mechanism, similar to that proposed by Cardew and Davey [3]

\footnotetext{
* Corresponding author. Fax: +343944872.
}

for some polymorphic transformations. Crystal growth experiments in a porous silica gel transport medium allows us to reproduce the conditions of phosgenite and cerussite crystallization in nature and monitor the transformation process. The development of the transformation is strongly controlled by the structural elements that both phases have in common, providing an interesting example of topotaxy.

Phosgenite structure shows $\mathrm{P} 4 / \mathrm{mbm}$ symmetry, with $a=8.160 \AA$, and $c=8.883 \AA$. In this structure, the $\mathrm{Pb}$ and $\mathrm{Cl}$ atoms define layers parallel to (001). These sheets are connected by $\mathrm{CO}_{3}$ groups. The carbonate groups have the usual shape and lie in $\{110\}$ mirror planes. Lead is coordinated by five $\mathrm{Cl}$ and four $\mathrm{O}$ atoms [4]. Cerussite shows orthorhombic aragonite-type structure. The space group is Pmcn, with $a=5.15 \AA, b=8.47 \AA$, and $c=6.11 \AA$ [5]. In this structure, the $\mathrm{Pb}$ atoms are pseudohexagonally 
arranged in layers parallel to (001). These layers are separated by "corrugated" layers of $\mathrm{CO}_{3}$ groups that also show a pseudohexagonal arrangement. Each $\mathrm{CO}_{3}$ group is surrounded by six $\mathrm{Pb}$ atoms, and each $\mathrm{Pb}$ atom has nine immediate oxygen neighbours.

In this paper, we deal with the topotactic transformation of phosgenite into cerussite. The observed topotactic relationships are interpreted on the grounds of structural considerations.

\section{Experimental procedure}

Growth experiments were carried out in a double-diffusion system as shown in Fig. 1. The horizontal branch is occupied by a column of polymerized silica hydrogel. The length and the diameter of the gel column were 280 and $90 \mathrm{~mm}$, respectively. The reservoirs $\mathrm{A}$ and $\mathrm{B}$ were filled with $7.5 \mathrm{~cm}^{3}$ of $\mathrm{Na}_{2} \mathrm{CO}_{3} 0.1 \mathrm{~N}$ and $\mathrm{PbCl}_{2} 0.009 \mathrm{M}$ mother solutions, respectively. Both reactants diffuse through the gel column. Silica gel was prepared by acidifying a sodium silicate solution (Merck, specific gravity $\left.1.059 \mathrm{~g} / \mathrm{cm}^{3}, \mathrm{pH}=11.2\right)$ to desired $\mathrm{pH}(5.5)$ by adding $\mathrm{HCl}(1 \mathrm{~N})$. All the experiments were carried out at $25^{\circ} \mathrm{C}$.

The crystallization sequence and the alterationtransformation phenomena were monitored by optical microscopy. After 8 months since nucleation occurred, the crystals were recovered by dissolving the silica gel in a $\mathrm{NaOH}$ solution (1M). Samples corresponding to the different phases and to different degrees of transformation were hand-picked. Their morphologies and transformation features were studied by means of scanning electron microscopy. The different phases obtained were identified by $\mathrm{X}$-ray powder diffraction.

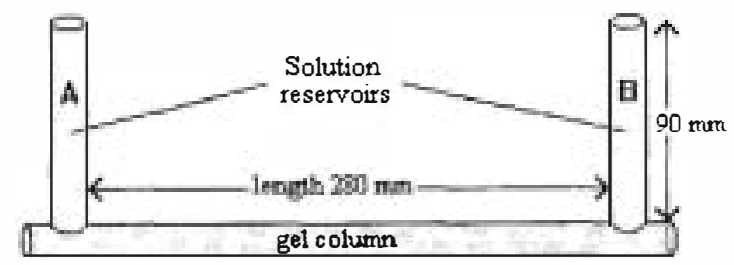

Fig. 1. Experimental set-up.

\section{Results}

The crystallization sequence observed starts with the nucleation of phosgenite crystals in the region of the gel column close to the $\mathrm{PbCl}_{2}$ reservoir. The nucleation density is very low $\left(1-2\right.$ nuclei $\left.\cdot \mathrm{cm}^{-3}\right)$. During the early stages of the growth process, phosgenite crystals show dendritic developments along $\langle 110\rangle$ directions. The most common forms present are $\{001\},\{100\},\{110\},\{111\}$, and $\{120\}$. Their relative development evolves during the growth process, leading to a more faceted crystal habit [6]. However, the final growth form always exhibits a polyhedral habit dominated by $\{001\}$ and $\{100\}$ prismatic faces. All the experimental growth forms found occupy the first places of the Donnay-Harker ranking for phosgenite: $001,101,110,111,020,200,102,021,201$, $120,210,112, \cdots$. The size of the crystals ranged from 250 to $500 \mu \mathrm{m}$.

As time passes by, phosgenite crystals develop signs of dissolution (dissolution pitches) and cerussite nucleates. In diffusing-reacting systems, such as the one we are dealing with, the advancement of mass transfer determines the development of concentration, $\mathrm{pH}$, and supersaturation gradients [7]. Both the morphological evolution and the crystallizationdissolution phenomena observed can be related to changes in the supersaturation degree during the growth process. However, a detailed study of the physical chemistry of the system is beyond the scope of this work. The nucleation of cerussite crystals preferentially occurs on the surface of phosgenite crystals and shows a clear epitactic character. Cerussite grows as thin needle-shaped single crystals elongated along the [001] direction. The length of these crystals, measured along [001], ranged from 30 to 60 $\mu \mathrm{m}$, while their width, measured along [100], ranged from 5 to $10 \mu \mathrm{m}$. The habit is dominated by $\{100\}$, $\{010\}$, and $\{110\}$ prismatic forms. Moreover, the crystals are terminated by $\{102\}$ and $\{001\}$, these forms showing scarce development. All the forms mentioned are important forms within the cerussite Donnay-Harker sequence: 110, 010, 111, 021, 001, 012, 102, 100, 112, 130, 221.

Phosgenite dissolution and cerussite epitactic heterogeneous nucleation represent the starting point for a phase transformation in which the two phases involved are separated by a thin solvent film. The 


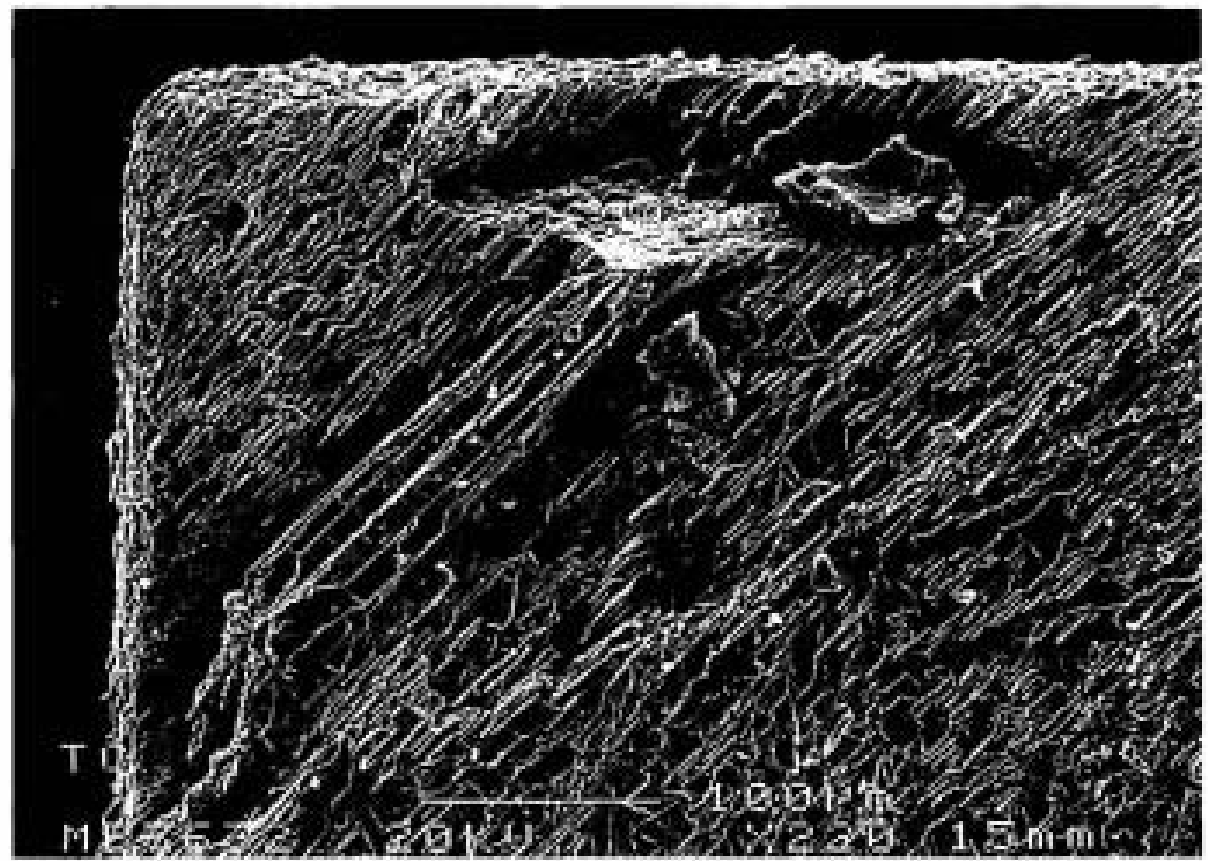

Fig. 2. SEM microphotograph of phosgenite pseudomorphized by cerussite aggregates.

transformation starts in the edges and comers of phosgenite crystals and, soon, an advancing front is defined. The solvent film between both phases al- lows the metastable phosgenite to dissolve while cerussite nucleates and grows independently from solution. Both processes are simultaneous and the

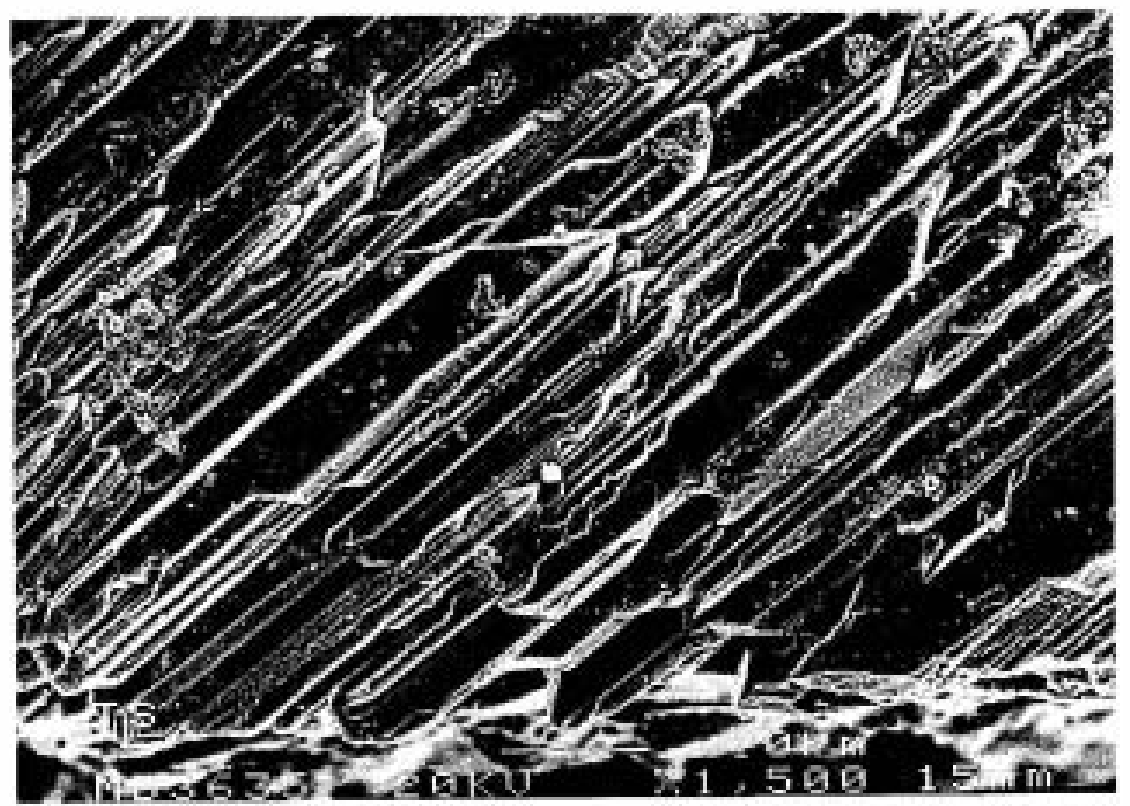

Fig. 3. Detail of the topotaxy. 


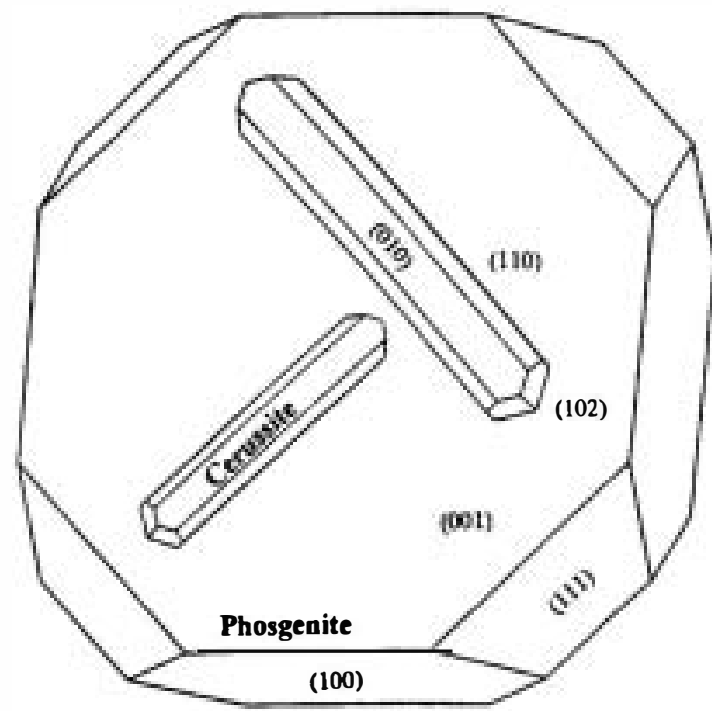

Fig. 4. Idealized representation showing the phosgenite-cerussite topotaxy relationships.

transformation proceeds through the bulk of the initial phase preserving the external shape of phosgenite crystals. The final pseudomorph of phosgenite is built up by aggregates of parallel crystals of cerussite elongated along [001].

Fig. 2 shows a phosgenite crystal that has been

\section{Phosgenite}

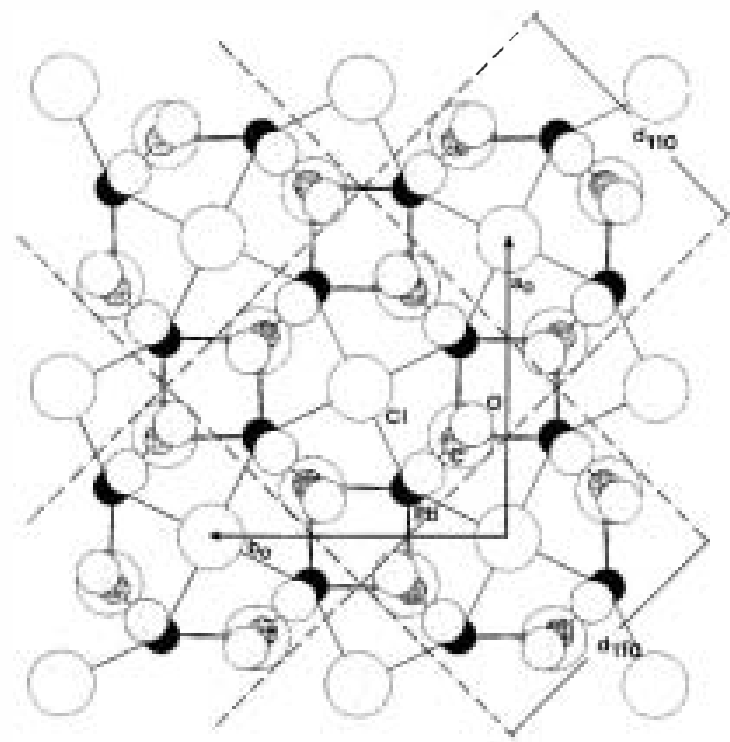

pseudomorphized by prismatic crystals of cerussite. The long axis of cerussite fibres form a $45^{\circ}$ angle with the [100] direction of the initial phosgenite crystal. All the characteristics observed indicate that we are dealing with a topotactic transformation [8]. The most evident feature is the parallelism between the $\langle 110\rangle$ direction of phosgenite and the $\langle 001\rangle$ direction of cerussite. A detailed view of the topotaxy cerussite-phosgenite can be observed in Fig. 3. The fact that there are two equivalent orientations of $\langle 110\rangle$ directions in phosgenite and only one [001] orientation in cerussite determines that in the transformation from phosgenite to cerussite, cerussite crystals may grow with two orthogonal orientations that may be twin-related, each parallel to one of the $\langle 110\rangle$ directions of phosgenite (Fig. 4).

\section{Discussion}

As we mentioned above, the heterogeneous nucleation of cerussite on the surfaces of phosgenite crystals represents the starting point of the topotactic transformation of phosgenite into cerussite. Frequently, cerussite crystals nucleate on the substrate showing a clear orientation. Von Vultee [9] described

\section{Cerussite}

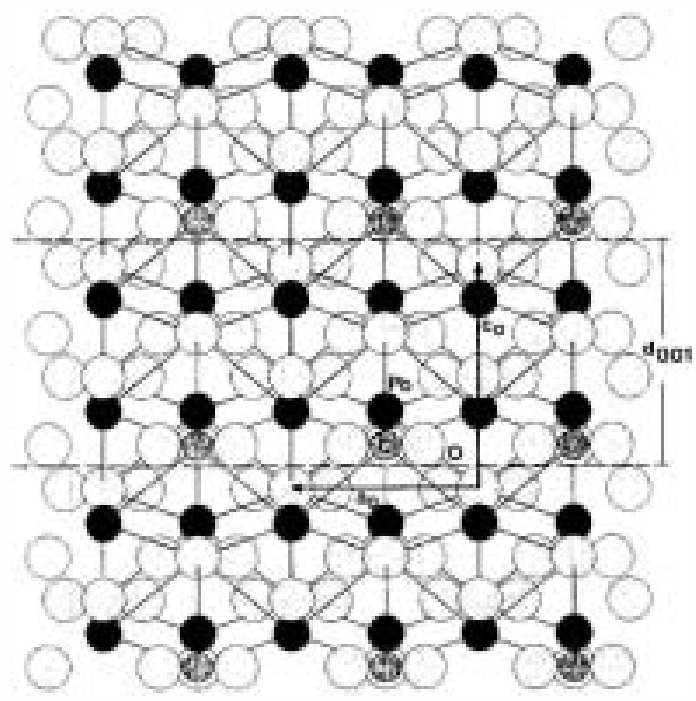

Fig. 5. Projection of phosgenite and cerussite structures on (001) and (010), respectively. 
the growth of cerussite on phosgenite as an epitaxy, where the main coincident pairs of crystallographic directions and planes are $\langle 110\rangle_{\mathrm{ph}} \|[001]_{\mathrm{cer}}$ and $(001)_{\mathrm{ph}} \|(010)_{\mathrm{cer}}$. It seems that this epitactic growth controls the subsequent development of the phosgenite-cerussite topotactic transformation.

Both in the development of epitactic overgrowth and topotactic transformations, the geometric relationships between the crystal lattice of the phases involved play a fundamental role. Moreover, in order to understand the topotaxy phenomenon, the structural elements that both phases have in common must be considered. In the case that we are dealing with, $\mathrm{CO}_{3}$ groups are the main element shared by both structures. A first comparison between the projection of phosgenite and cerussite structures on (001) and (010), respectively, shows the similarity of the distribution of $\mathrm{CO}_{3}$ groups in both phases (Fig. 5). In phosgenite structure $\mathrm{CO}_{3}$ groups define layers parallel to $\{110\}$, while in cerussite structure these groups are arranged in layers parallel to (001). The development of the topotaxy determines the parallelism of these layers in the two structures. For phosgenite the distance between successive $\mathrm{CO}_{3}$ groups along $\langle 110\rangle$ directions is $5.77 \AA$, a half of the translation period along those directions. In the case of cerussite, the distance between successive $\mathrm{CO}_{3}$ groups along the [001] direction is $6.13 \AA$, i.e., it coincides with the $c_{0}$ parameter. When a $1: 2$ ratio between the repeating periods along $\langle 110\rangle_{\mathrm{ph}}$ and $[001]_{\text {cer }}$ directions is considered, the calculated linear misfit is $6.33 \%$. Moreover, the value of the distance between successive $\mathrm{CO}_{3}$ groups along the [100] direction in the cerussite structure is $5.18 \AA$, i.e. coinciding with the $a_{0}$ parameter. In this case, the misfit between the two parallel directions in both structures $\left(\langle 110\rangle_{\mathrm{ph}}\right.$ and $\left.[100]_{\mathrm{cer}}\right)$ is $11.37 \%$. Finally, along the third orthogonal direction, [001] in phosgenite and [010] in cerussite, the repeating periods are 8.88 and $8.49 \AA$, respectively, giving a linear misfit of $4.60 \%$. Table 1 shows a comparison between the translational periods along the directions involved in the topotaxy. The reduced misfits between pairs of parallel directions explain the good tridimensional matching between both structures. The close dimensional correspondence found between the strain-free lattices at the phase boundary guarantees the minimization of the interfacial energy [10].
Table 1

Comparison between topotaxy directions

\begin{tabular}{lcl}
\hline $\begin{array}{l}\text { Phosgenite } \\
\mathrm{Pb}_{2} \mathrm{Cl}_{2} \mathrm{CO}_{3}\end{array}$ & $\begin{array}{l}\text { Cerussite } \\
\mathrm{PbCO}_{3}\end{array}$ \\
\hline Parameter $(\AA)$ & Misfit $(\%)$ & Parameter $(\AA)$ \\
\hline $2 \times\langle 110\rangle=5.77$ & 6.33 & {$[001]=6.13$} \\
$2 \times\langle 110\rangle=5.77$ & 11.37 & {$[100]=5.18$} \\
{$[001]=8.88$} & 4.60 & {$[010]=8.49$} \\
\hline
\end{tabular}

Moreover, this matching favours the oriented heterogenous nucleation of cerussite on $\{001\}$ surfaces of phosgenite crystals. As it has been mentioned above, this phenomenon precedes and is in the origin of the topotactic transformation of phosgenite into cerussite.

Apart from the geometrical relationships between both lattices, other structural features may play an important role in the development of epitactic-topotactic phenomena. The coincidence between periodic bond chains (PBCs) [11] in phosgenite and cerussite structures is an interesting aspect to be taken into account. Fig. 5 shows a comparison between the structures of phosgenite and cerussite, projected along [001] and [010], respectively. In the case of phosgenite structure, $\mathrm{Pb}-\left(\mathrm{Cl}, \mathrm{CO}_{3}\right)-\mathrm{Pb}$ chains along $\langle 110\rangle$ and $\mathrm{Pb}-\mathrm{Cl}-\mathrm{Pb}-\mathrm{CO}_{3}-\mathrm{Pb}$ chains along [001] can be observed. The presence of these chains gives a strong $F$ character to $\{110\}$ and $(001)$ faces. In the same way, in the cerussite structure it is possible to define $\mathrm{Pb}-\mathrm{CO}_{3}-\mathrm{Pb}$ chains along [001] and<smiles>O=C(O)OC(=O)[PH](=O)c1ccccc1</smiles>

chains along [100], [110] and [1피. Thus, (001), (010) and (110) are F faces [12]. It seems clear that an important role has been played by $F$ faces and strong cation-anion bonds during the transformation. Hence, the topotactically related faces are $F$ faces and the topotaxy directions correspond to $\mathrm{PBC}$ vectors. In the case of $\langle 110\rangle$ directions in phosgenite structure, these PBCs are built by $\mathrm{Pb}-\left(\mathrm{Cl}, \mathrm{CO}_{3}\right)-\mathrm{Pb}$ chains, while along the [001] direction of cerussite there are simple chains $\mathrm{Pb}-\mathrm{CO}_{3}-\mathrm{Pb}$. It can be concluded that the presence of these primitive PBCs structurally controls the transformation of phosgenite into cerussite preserving the strong $\mathrm{Pb}-\mathrm{CO}_{3}$ bonds. 
Moreover, according to Giuseppetti and Tadini [4], the $\mathrm{Pb}-\mathrm{Cl}$ bonds are weak ionic bonds. This feature favours the release of $\mathrm{Cl}$ atoms required by the transformation.

\section{Conclusions}

(1) The crystallization of phosgenite and cerussite was carried out in a diffusing-reacting system. The crystallization sequence starts with the nucleation and growth of phosgenite crystals. The physicochemical evolution of the system determines that phosgenite crystals develop dissolution signs and cerussite nucleates.

(2) Phosgenite crystals undergo a morphological evolution as a consequence of the supersaturation decrease. Its final growth morphology is defined by $\{001\},\{100\},\{110\},\{111\}$, and $\{120\}$. Cerussite habit is dominated by $\{100\},\{010\}$, and $\{110\}$, while $\{102\}$ and $\{001\}$ show a scarce development. In both cases, the growth morphology is defined by important forms according to the Donnay-Harker method.

(3) Nucleation of cerussite occurs epitaxially on the surface of phosgenite crystals. It represents the starting point for the subsequent development of the transformation of phosgenite into cerussite. This transformation shows a clear topotactic character. The main orientation relations that have been found are the parallelism between $\langle 110\rangle_{\mathrm{ph}}$ and $[001]_{\mathrm{cer}}$, and $\langle 110\rangle_{\mathrm{ph}}$ and $[100]_{\mathrm{cer}}$, The most frequent related planes are $(001)_{\mathrm{ph}}$ and $(010)_{\mathrm{cer}}$. The reduced misfits found between pairs of parallel directions explains the good tridimensional matching between both structures.
(4) The faces topotactically related are F faces and the topotaxy relations correspond to $\mathrm{PBC}$ vectors. The presence of these primitive PBCs structurally controls the transformation of phosgenite into cerussite. During the transformation process, $\mathrm{Pb}-\mathrm{CO}_{3}$ bonds are preserved, while the weakness of $\mathrm{Pb}-\mathrm{Cl}$ bonds favours the release of $\mathrm{Cl}$ atoms.

\section{Acknowledgements}

This work was supported by DGCYT (Grant PB92-0998).

\section{References}

[1] R. Edwards, R.D. Gillard, P.A. Williams and A.M. Pollard, Mineral. Mag. 56 (1992) 53.

[2] G. Rossi, I. Uras, R. Valera, Rend. Soc. Ital. Mineral. Petr. 29 (1973) 219.

[3] T.T. Cardew and R.J. Davey, Proc. R. Soc. London A 398 (1985) 415.

[4] G. Giuseppetti and C. Tadini, Tschermaks Mineral. Petr. Mitt. 21 (1974) 101.

[5] G.M. Lindsay and H.C. Hoyt, Z. Krist. 100 (1939) 360.

[6] C.M. Pina, M. Prieto and L. Femández-Díaz, Bol. Soc. Esp. Min. 15 (1) (1982) 11.

[7] M. Prieto, L. Femández-Diaz and S. López-Andrés, J. Crystal Growth 108 (1991) 770.

[8] C.N.R. Rao and K.J. Rao, Phase Transition in Solids (McGraw-Hill, New York, 1978).

[9] J. von Vultée, Fortschrift Mineral. 29/39 (1950) 297.

[10] M.E. Fleet, Am. Mineral. 67 (1982) 926.

[11] P. Hartman, in: Morphology of Crystals, Part A, Ed I. Sunagawa (Terra, Tokyo, 1987) ch. 4.

[12] W. Franke, K. Lenk, M.A. Ittyachen and K.J. Mohanan Pillai, J. Crystal Growth 51 (1981) 309. 